\title{
TP53 mutations in early-stage ovarian carcinoma, relation to long-term survival
}

\section{Y Wang ${ }^{1,2}$, Å Helland ${ }^{2,3}$, R Holm $^{4}$, H Skomedal $^{4}$, VM Abeler $^{4}$, HE Danielsen ${ }^{4}$, CG Tropé', A-L Børresen-Dale $^{2}$ and GB Kristensen ${ }^{*}, 1$}

'Department of Gynecologic Oncology, The Norwegian Radium Hospital, Oslo N-03 I0, Norway; ${ }^{2}$ Department of Genetics, The Norwegian Radium Hospital, Oslo N-0310, Norway; ${ }^{3}$ Department of Oncology, The Norwegian Radium Hospital, Oslo N-0310, Norway; ${ }^{4}$ Department of Pathology, The Norwegian Radium Hospital, Oslo N-0310, Norway

We conducted the present study to evaluate the frequency and prognostic importance on long-term survival of TP53 mutations and TP53 protein accumulation in a cohort of 178 patients with early-stage ovarian carcinomas. TP53 mutations scored as aberrant temporal temperature gradient gel electrophoresis pattern from all exons were observed in 39.9\% of the tumours. Full screening of exons 5-8, followed by sequencing, was successful in 135 cases, and 48 mutations altering the protein were detected in 39 cases (28.9\%). TP53 mutations were slightly less common in the Federation of Gynecologists and Obstetricians stage IA than in IB/IC $(P=0.05)$. No significant correlations with histological type, grade of differentiation, DNA ploidy status or age at diagnosis were found. TP53 protein accumulation analysed by immunohistochemistry was found in $32.6 \%$ of all tumours, and was a poor predictor of TP53 mutations with $56.4 \%$ sensitivity, $77.1 \%$ specificity, $50 \%$ positive predictive value and $81.3 \%$ negative predictive value. Neither TP53 mutations nor TP53 protein accumulation influenced the prognosis significantly in this group of patients.

British Journal of Cancer (2004) 90, 678-685. doi:I0.1038/sj.bjc.660I537 www.bjcancer.com

(c) 2004 Cancer Research UK

Keywords: TP53 tumour-suppressor gene; TP53 mutation; TP53 protein accumulation; ovarian carcinoma; prognosis

Ovarian cancer is associated with the highest mortality rate among gynecologic malignancies. The ovaries have a relatively inaccessible location and ovarian cancer patients very often lack symptoms in early stages. Around $70 \%$ of the cases are diagnosed at advanced stages of the disease, with either regional or distant metastasis. No efficient screening methods are available and our understanding of the underlying biology of the disease is still sparse. For patients diagnosed in Norway between 1989 and 1993 the 5 -year relative survival rate was $37 \%$ for all cases and $78 \%$ for patients diagnosed with early stages of this disease (Bjorge et al, 1998). Despite progress in surgical and chemotherapy treatment, no major improvement in the long-term survival has been achieved. The genetic mechanisms contributing to the development and progression of epithelial ovarian cancer are not well understood, and the lack of good prognostic markers has led to several studies aiming at identifying new molecular markers with both predictive and prognostic value.

The TP53 tumour-suppressor gene is frequently mutated in human cancer, and has been shown to be a prognostic marker in several tumour types (Hollstein et al, 1991; Borresen-Dale, 2003; Iacopetta, 2003). The gene is located on the short arm of chromosome 17, at position $17 \mathrm{p} 13.1$, and encodes a $53 \mathrm{kDa}$ nuclear phosphoprotein whose regulatory actions include both the inhibition and stimulation of transcription (Levine et al, 1991). The TP53 protein acts as a checkpoint control for recognising

* Correspondence: GB Kristensen;

E-mail: gunnar.kristensen@klinmed.uio.no

Revised 9 September 2003; accepted 5 November 2003 damaged DNA, allowing DNA repair and delayed entrance into the DNA-replication phase of the cell cycle. When DNA repair is not possible, programmed cell death or apoptosis follows. The loss of tumour-suppressor function of the TP53 protein, subsequent to a mutation in the coding sequence, seems to be a feature common to many cancers, including ovarian cancer. The primary mechanism of this dysfunction in ovarian cancer is a missense mutation in the evolutionary conserved domains of the gene. However, microdeletions, insertions and nonsense mutations have also been described (Sood et al, 1999; Buller et al, 2001). A number of studies have analysed ovarian carcinomas for TP53 mutations, but mostly in advanced stages (Kohler et al, 1993; Kupryjanczyk et al, 1993, 1995; Fallows et al, 2001; Reles et al, 2001; Leitao et al, 2002). The frequency of mutations was reported to be in the range of $29-79 \%$ depending on the stage of the disease, with the highest frequency found in tumours from patients diagnosed with late-stage disease.

The impact of TP53 alterations on prognosis in patients with ovarian cancer has also been studied, but again mainly focused on advanced disease. The majority of studies demonstrated that TP53 alterations failed to influence the prognosis in multivariate analysis including known prognostic factors (Wen et al, 1999; Schildkraut et al, 2000; Fallows et al, 2001). Only few studies have focused on the early stage of ovarian carcinomas, and these have indicated that TP53 alteration may be an early genetic event occurring before metastasis (Kohler et al, 1993; Kupryjanczyk et al, 1995). The number of samples studied has, however, been limited and the follow-up time relatively short. This study reports TP53 mutations and TP53 protein expression among 178 patients with early-stage ovarian carcinomas, in order to evaluate the prognostic significance of TP53 alterations on long-term survival. 


\section{MATERIALS AND METHODS}

\section{Patient population and clinical data}

The study population consisted of a total of 178 patients with early-stage ovarian carcinomas, who were treated at the Department of Gynecological Oncology at The Norwegian Radium Hospital (NRH) during the period 1982-1989. Generally, surgery was performed at county hospitals and the patients were admitted to NRH for evaluation of further treatment. The surgical procedure consisted of a midline incision with peritoneal washing, hysterectomy, bilateral salpingo-oophorectomy, omentectomy and throughout inspection of the abdominal cavity. Lymph node sampling was not performed. In all patients, the tumours were restricted to one or both ovaries by these evaluations, and thereby apparently in the International Federation of Gynecologists and Obstetricians (FIGO) stage I. All tissue removed at surgery was immediately fixated in buffered formalin and processed at the Department for Pathology within 24-72 h. One single pathologist at NRH reviewed all histological sections. The histological type and grade of differentiation were based on World Health Organization criteria. All, but eight patients, had adjuvant treatment following surgery, 90 had chemotherapy (76 cisplatinum as a single drug and 14 thiotepa), 63 had intraperitoneal instillation of ${ }^{32} \mathrm{P}, 15$ had whole abdominal external radiation, while two patients had other types of treatment.

All patients have been followed up at the NRH or at local hospitals until death or December 31, 1999. Follow-up of patients alive without relapse ranged from 10 to 17 years, with a median follow-up of 13.9 years. Patient's age ranged from 15 to 83 years, with a median age of 57.8 years. The clinical and histopathological characteristics of the patients are summarised in Table 1. DNA ploidy analysis was performed with a high-resolution image system, as previously described (Sudbo et al, 2001).

\section{Mutation analyses}

Haematoxylin-Eosin-stained sections were used to evaluate the approximate percentage of tumour tissue. All samples contained $20 \%$ or more tumour tissue. DNA was isolated from paraffinembedded ovarian carcinoma tissues by phenol/chloroform extraction, followed by ethanol precipitation. Amplification of exons $2-11$ of the TP53 gene was performed by the polymerase chain reaction (PCR), followed by temporal temperature gradient gel electrophoresis (TTGE) analyses. All samples with aberrantly migrating bands in exons 5-8 were submitted to direct sequencing of a new PCR product using standard dideoxy sequencing reaction and the Dye Terminator Cycle Sequencing kit with AmpliTaq FS, and analysed on an ABI 373 sequencer (Applied Biosystems). The primer sets and condition for TTGE and sequencing were as previously described (Sørlie et al, 2003).

\section{Immunohistochemistry}

Formalin-fixed, paraffin-embedded tissue specimens were used for immunohistochemical staining with the avidin - biotin - peroxidase complex (ABC) method, as previously described (Hsu et al, 1981).

Table I Clinicopathologic parameters in relation to TP53 status

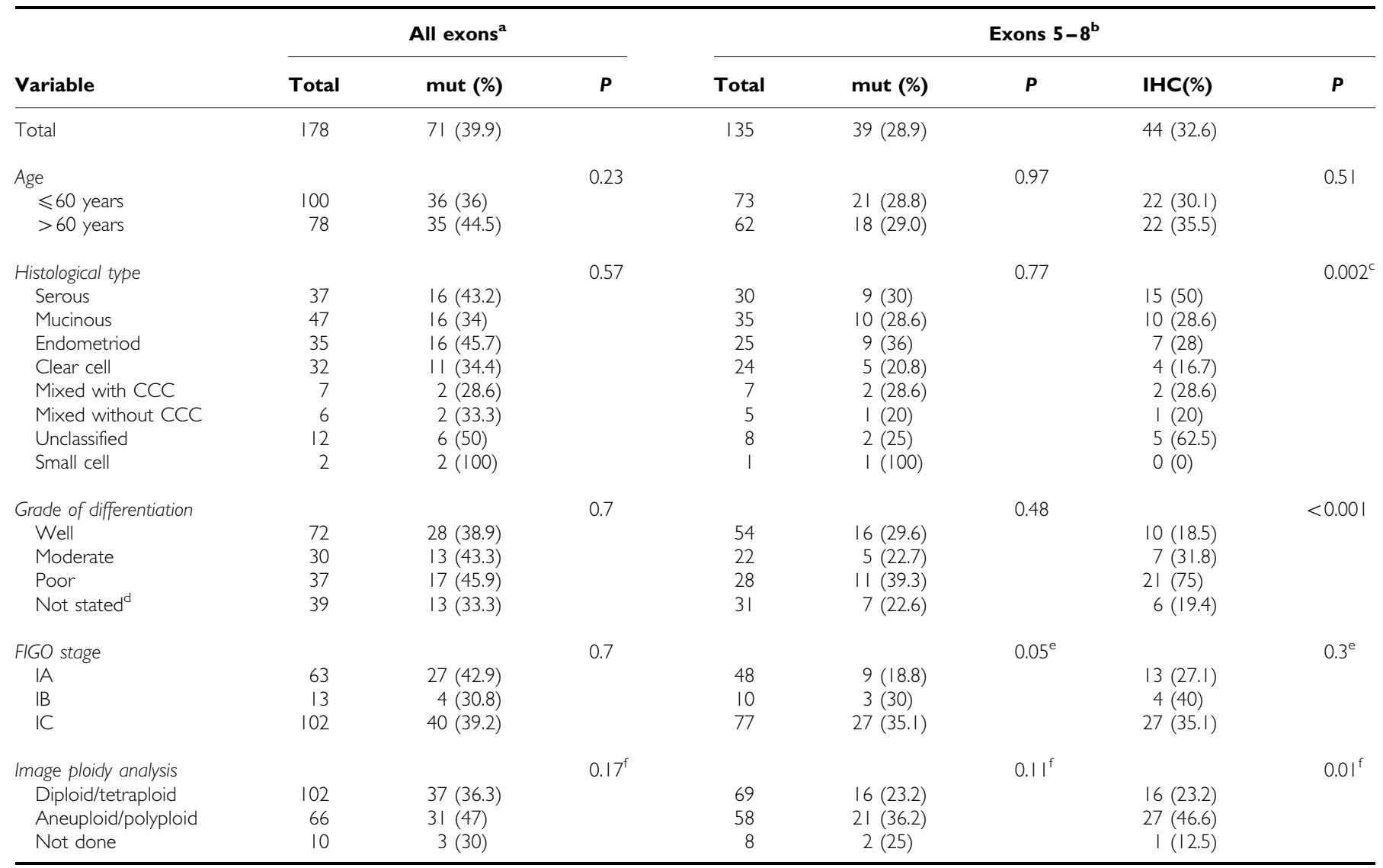

$\mathrm{CCC}=$ clear cell component; mut $=$ TP53 mutation; IHC $=$ TP53 protein accumulation by IHC. ${ }^{\mathrm{a}}$ Mutations scored as aberrant by TTGE shifts. ${ }^{\mathrm{b}}$ Only samples where exons $5-8$ were successfully analysed and mutations scored as sequence alterations leading to altered protein were included. ${ }^{\mathrm{C}} \mathrm{Serous}$ and unclassified compared with all other types. ${ }^{\mathrm{d}} \mathrm{G}$ rade of differentiation was not stated for the clear cell carcinoma or for mixed tumours with clear cell components. ${ }^{e} \mathrm{FIGO}$ stage IA compared with stages IB and IC. fDiploid/tetraploid compared with aneuploid/poliploid. 
The sections were microwave-treated in $10 \mathrm{~mm}$ citrate buffer, $\mathrm{pH}$ 6.0 , to unmask the epitopes of TP53 proteins. Then, the sections were incubated for $18-22 \mathrm{~h}$ in $4^{\circ} \mathrm{C}$ with the polyclonal TP53 antiserum (NCL-CM1, Novocastra laboratory Ltd, UK), diluted 1:2000, and monoclonal antibodies TP53 (DO-1, Santa Cruz Biotechnology Inc., CA, USA) diluted 1:1000 (1 $\left.\mu \mathrm{g} \mathrm{IGg}_{2 \mathrm{a}} \mathrm{ml}^{-1}\right)$. Both TP53 antibodies detected mutant and wild-type TP53 protein. The sections were incubated with a biotin-labelled secondary antibody and avidin-biotin-peroxidase complex. The peroxidase reaction was developed using diaminobenzidine as chromogen. All series included replacement of polyclonal primary antiserum with normal rabbit serum diluted $1: 2000$, whereas negative controls for the monoclonal antibody were performed using mouse myeloma protein of the same subclass and concentration as the monoclonal antibody. All controls gave satisfactory results. The results of the immunohistochemical analysis were interpreted without knowledge of the clinical information or mutation status using TTGE analyses. Three semiquantitative classes were used to describe the number of immunostained cells: - , none or less than $10 \%$ of the cells;,$+ 10-50 \%$ of the cancer cells; and ++ , more than $50 \%$ of the cells. The tumours with more than $10 \%$ immunostained cells in either of the two TP53 antibodies (NCL-CM1 and DO-1) were defined to have TP53 protein accumulation. A section from a breast carcinoma with TP53 mutation was used as a positive immunohistochemical control.

\section{Statistics}

Differences in proportions were evaluated by the $\chi^{2}$ test. Diseasefree survival was defined as the time interval between diagnosis and relapse of ovarian cancer. The method of Kaplan and Meier and the log-rank test were used to estimate and compare survival rates. Independent prognostic factors were determined by Cox proportion hazards analysis. Statistical significance was considered as $P<0.05$.

\section{RESULTS}

\section{TP53 mutation frequency}

A total of 178 primary early-stage ovarian carcinomas were analysed for TP53 mutations in exons 2-11 using the TTGE methodology. Mutations scored as an aberrant TTGE pattern were seen in 71 cases $(39.9 \%)$, with $75 \%$ of the mutations located in exons 5-8. Several samples had more than one mutation and a total of 91 aberrant TTGE patterns were observed. This frequency may be an underestimate, since not all exons gave a PCR product that could be analysed by TTGE. Success rate in obtaining a clean and analysable PCR product ranged from 25.3 to $97.8 \%$, with the lowest success rate for exons 4 and 10. Samples with mutations found in exons $2-4$ and $9-11$ by TTGE were not submitted to sequencing, due to limited amount of mutations in most of these exons and problems with PCR amplification due to the limited amount of DNA. Exons 5-8 were successfully analysed for a total of 135 samples by TTGE, followed by sequencing of all samples with aberrant migrating bands on TTGE, and a total of 65 TP53 alterations were detected in 53 out of 135 (39.3\%) samples by TTGE.

\section{Spectrum of TP53 mutations in exons $5-8$ by direct sequencing}

The exact sequence alterations were successfully determined by direct sequencing in 59 of the cases representing 47 different patients (Table 2). An example of a TTGE analysis followed by sequencing is shown in Figure 1A-C. Of the 59 mutations detected, nine were silent mutations, with none being common polymorphisms. Two were intronic mutations with unknown function, leaving a total of 48 mutations altering the TP53 protein in 39 different patients $(28.9 \%)$. Seven patients revealed two different mutations and one had three different mutations. The mutations were distributed throughout the four exons examined, with 21 in exon 5, nine in exon 6,17 in exon 7 and 18 in exon 8 (Figure $2 \mathrm{~A}$ and $\mathrm{B}$ ). The majority of the sequenced mutations in this region, 56 of $59(94.9 \%)$, was point mutations. In all, $44(75 \%)$ of the mutations were missense with one sample having two base changes in the same codon (No. 308). Two samples had a deletion consisting of the same single base pair loss (no. 329; no. 378), leading to a frameshift mutation, and one sample (no. 363) had a 12 base pair deletion in exon 5 , leading to an in-frame mutation. The majority of the point mutations appeared at G:C base pairs (46 of $56,82.1 \%$ ), of which 11 were at $\mathrm{CpG}$ dinucleotides, and $\mathrm{G}: \mathrm{C} \rightarrow \mathrm{A}: \mathrm{T}$ transitions accounted for $64.3 \%$ (36 of 56 ). Totally, there were $75 \%$ transitions and $25 \%$ transversions. In all, 17 of the mutations disrupted the loop 2 or loop 3 domains of TP53 protein. Mutations in codons 146, 152, 187, 200, 204, 225 and 301 have not previously been reported in the International Agency for Research on Cancer (IARC) TP53 mutation database (http://www.iarc.fr/ P53/index.html.2002) among ovarian cancers. The comparisons of exon and codon distributions with IARC TP53 mutation database are shown in Figure 2A and B.

\section{Immunohistochemistry}

Considering the 135 samples where all exons 5-8 were successfully analysed by TTGE followed by sequencing, 44 samples $(32.6 \%)$ demonstrated TP53 protein accumulation by IHC, with 27 samples showing immunostaining in more than 50 percent of the tumour cells and 17 samples in $10-50 \%$ of the cells. The correlation between TP53 protein accumulation and TP53 mutations was significant $(P<0.001)$; however, the concordance was only $71.1 \%$ (96 of 135). The sensitivity, specificity, positive and negative predictive values of IHC analysis of TP53 protein accumulation in predicting for TP53 mutations were 56.4, 77.1, 50 and $81.3 \%$, respectively.

\section{TP53 mutations and clinicopathological parameters}

The relationship between TP53 mutations and clinicopathological parameters is shown in Table 1. TP53 mutation status was not significantly correlated with age at diagnosis, histological type, grade of differentiation or DNA ploidy status. However, there seemed to be a trend towards TP53 mutations being less common in FIGO stage IA than in IB/IC $(P=0.05)$. TP53 protein accumulation was significantly correlated with the grade of differentiation and DNA ploidy status, but not with FIGO stage or age at diagnose. TP53 protein accumulation was significantly more common in serous and unclassified than in other histological types (Table 1).

\section{Relationship between TP53 mutations and survival}

Kaplan-Meier analyses of disease-free survival in all 178 patients showed no difference between patients with and without TP53 mutations scored as a TTGE shift, the 5 -year survival rates being 66 and $81 \%$, respectively $(P=0.1)$. Furthermore, no significant difference was found between patients with or without TP53 protein accumulation by IHC, the 5-year survival rates being 69 and $78 \%$, respectively $(P=0.2)$. When considering the 135 samples with complete analyses of exons 5-8, there was no significant difference in disease-free survival between patients with or without TP53 mutations affecting the protein (Figure 3A). When samples with mutations affecting the L2/L3 loop of the protein were grouped separately, there was still no difference in survival rates (Figure 3B). When considering TP53 mutations and TP53 protein accumulation as TP53 aberrances, the 5-year survival rate was $80 \%$ 
Table 2 Characterization of the TP53 alterations found in exons 5-8 in this series

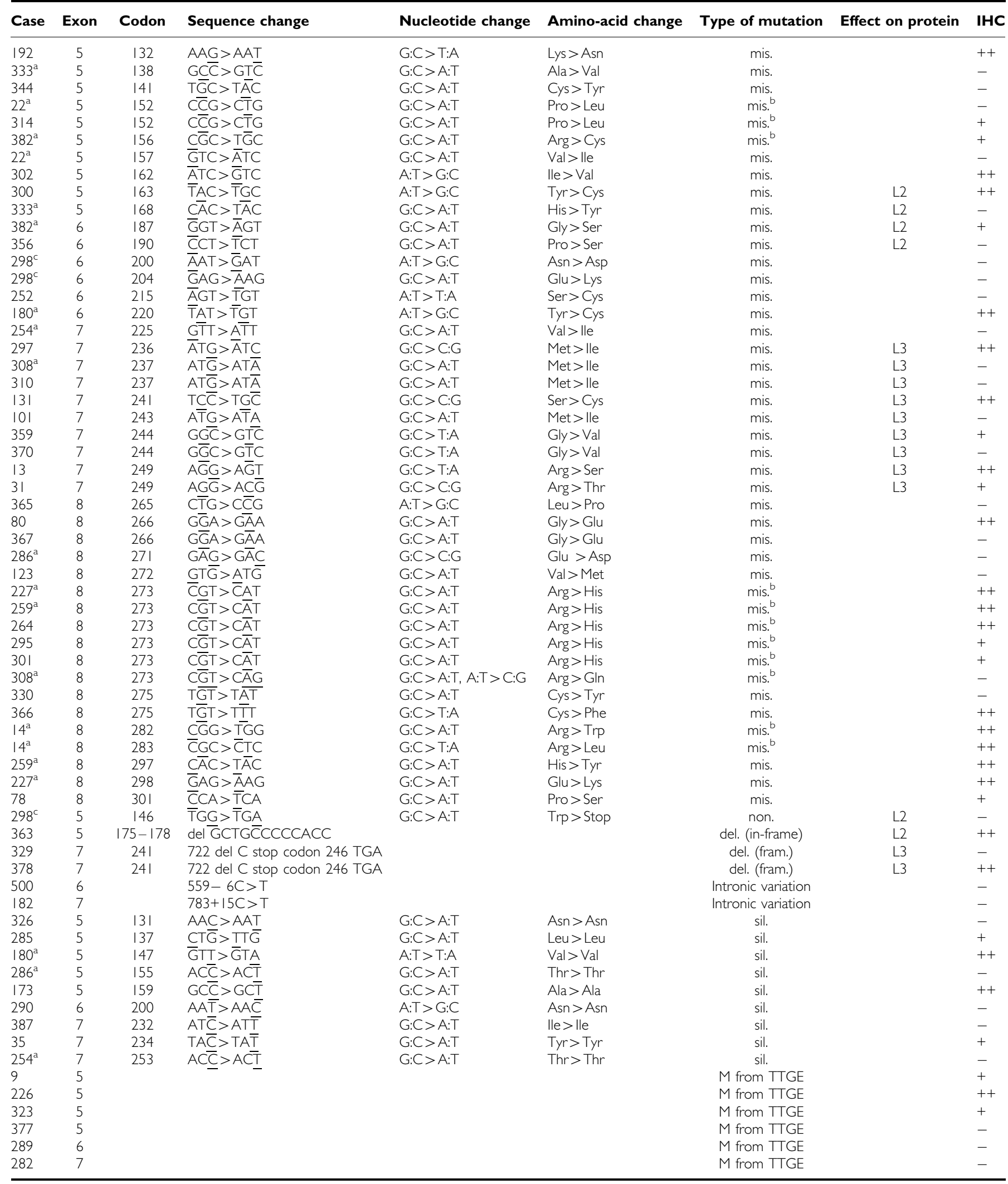

mis. = missense; sil. = silent mutation; non. = nonsense mutation; fram. = frameshift mutation; del. = deletion; $M$ from TTGE = mutation from TTGE, not enough high-quality DNA for sequence analysis. IHC: TP53 protein accumulation; - , no or $<10 \%$ cells with TP53 protein accumulation;,$+ 10-50 \%$ and $++>50 \%$ cells with TP53 protein accumulation. Effect on protein: effect of TP53 mutations on TP53 protein function. L2, L3: TP53 mutations affecting the loop 2 or loop 3 domains of the protein. ${ }^{2}$ Two mutations in the same sample. ${ }^{b} \mathrm{G}: \mathrm{C}>\mathrm{A}: \mathrm{T}$ transition at $\mathrm{CpG}$ dinucleotide. ${ }^{\mathrm{C}}$ Three mutations in the same sample. 
A

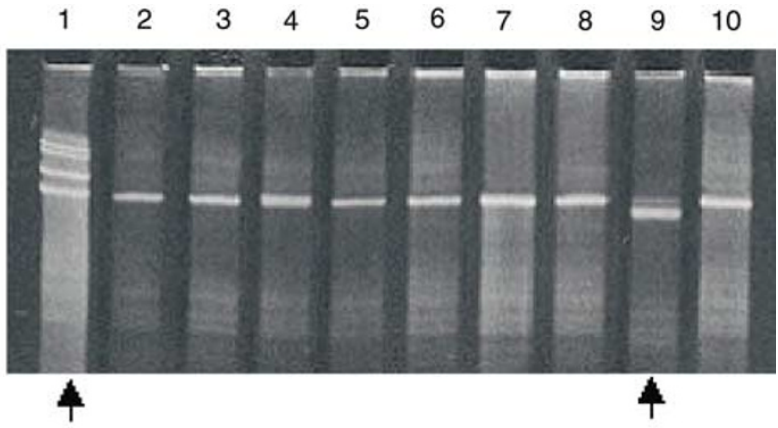

B

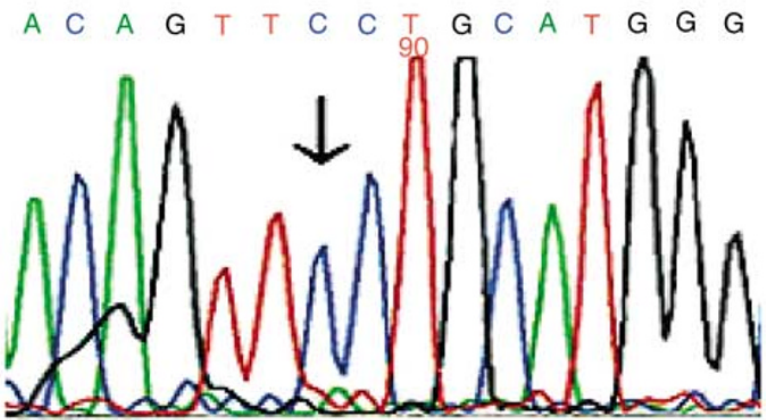

C
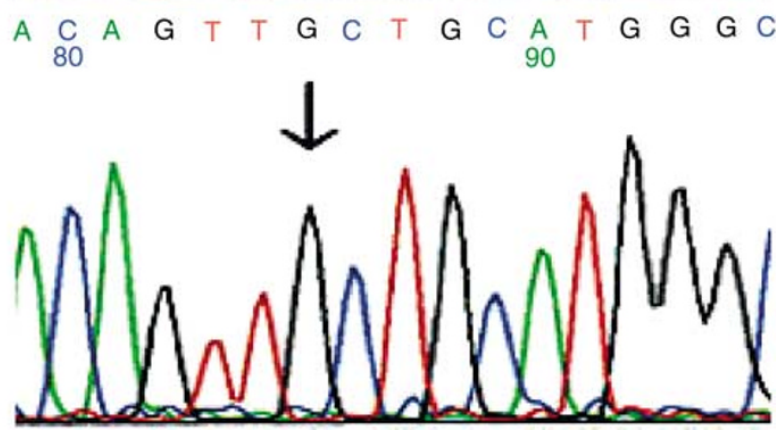

Figure I Temporal temperature gradient gel electrophoresis and sequence analysis on sample No. 131 with a $C$ to $G$ substitution at codon $24 \mathrm{I}$ in exon 7. (A) TTGE analyses with a mutation control in lane I and sample 131 in lane 9. (B) Direct sequencing result of a normal control. (C) Direct sequencing result of sample No. I3।.

for patients with wild type and $61 \%$ for patients with TP53 aberrance, but the difference in disease-free survival was not statistically significant (Figure 3C).

Among these 135 patients, platinum-based adjuvant chemotherapy was given to 59 patients and adjuvant radiotherapy to 57 patients after primary operation. Analysing these two patient groups separately did not give any difference in disease-free survival between patients with or without TP53 mutations in any of these groups.

\section{DISCUSSION}

TP53 has proved itself to be an important tumour-suppressor gene frequently found inactivated in human cancers, including ovarian carcinoma. We have analysed a large series of early-stage primary ovarian carcinomas, and found TP53 mutations in $39.9 \%$ of the tumours with $75 \%$ of the mutations located in exons $5-8$. The analysed cohort is unique, since it is a sizable number of earlystage ovarian carcinomas with long-term follow-up. Some variations may be expected in sampling procedures, since the tumour materials have been collected from different hospitals. However, the time from fixation until the tissue reached the pathology lab
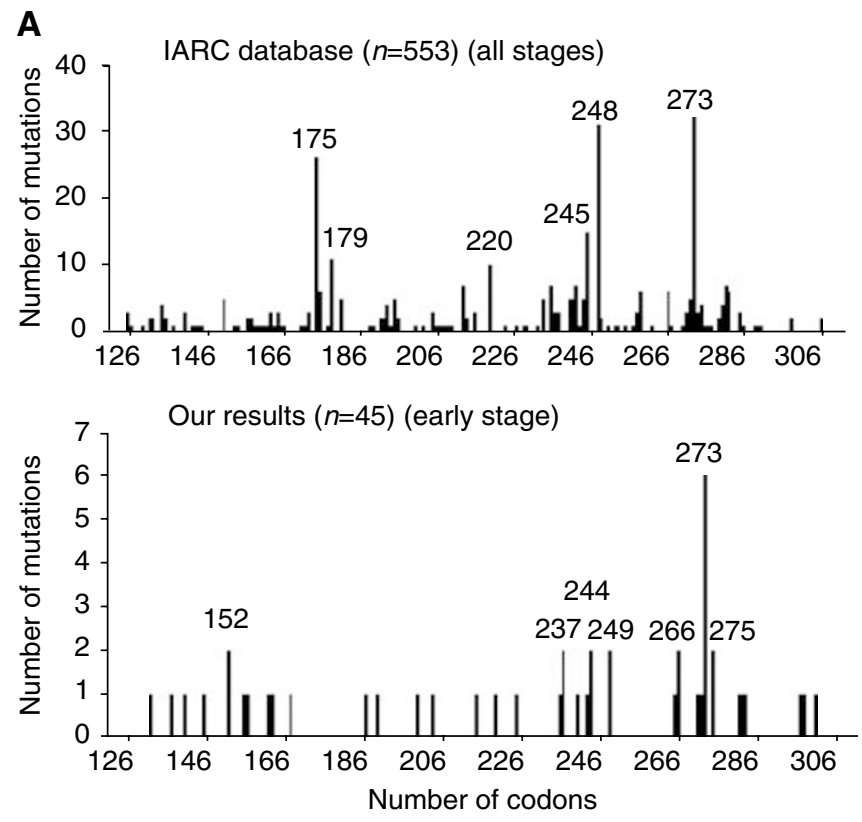

B

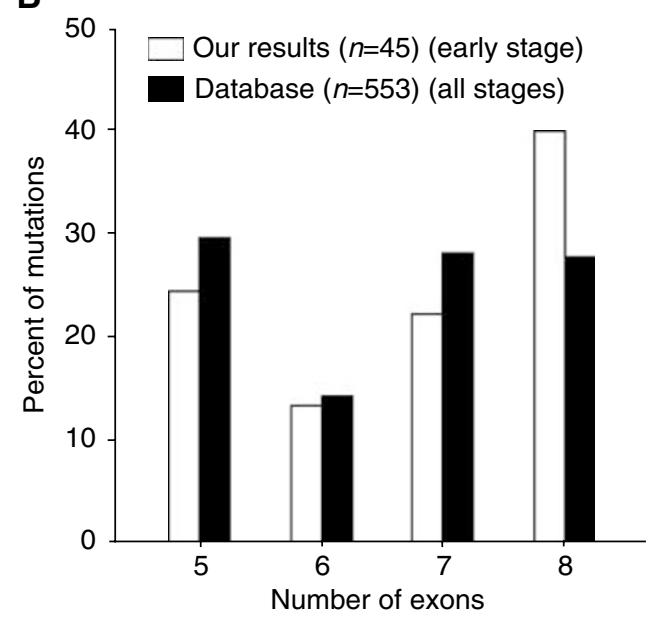

Figure 2 (A) Codon distribution of TP53 point mutations altering the TP53 protein. (B) Exon distribution of TP53 point mutations altering the TP53 protein (in percentage).

was less than $72 \mathrm{~h}$, and we considered this to be an acceptable uniform handling of the tissue. All tumour samples were obtained from primary operation before exposure to adjuvant therapy, thus avoiding any possible contribution from exposure to DNAdamaging agents. Among the 135 samples where exons 5-8 were successfully analysed, $39.3 \%$ revealed TP53 mutations. This result agrees with previous studies on smaller cohorts (Kohler et al, 1993; Buttitta et al, 1997; Reles et al, 2001; Leitao et al, 2002), which have shown a TP53 mutation rate in early-stage ovarian cancer between 15 and $50 \%$. In the present study, TTGE was used, followed by DNA direct sequencing to analyse TP53 mutations. Temporal temperature gradient gel electrophoresis is an improved mutationscreening technique (Borresen-Dale, 1996; Sørlie et al, 2003), which combines some advantages of the related techniques denaturing gradient gel electrophoresis (DGGE) and constant denaturant gel electrophoresis (CDGE), and eliminates some of the problems. The sensitivity of TTGE in detecting mutations present in only a small fraction of the sample is around $10 \%$ at the homoduplex level and $1-3 \%$ at the heteroduplex level, which is considerably better than can be achieved by direct sequencing. 
A

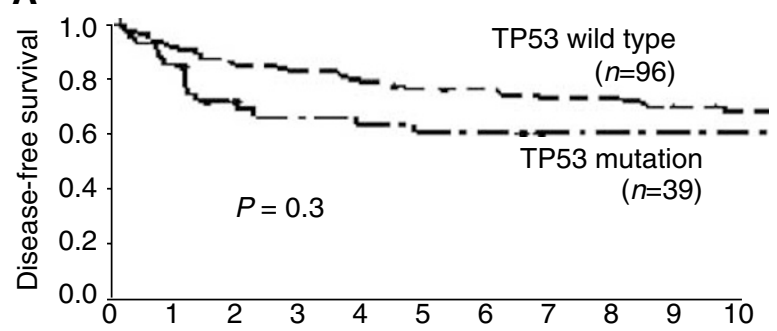

B

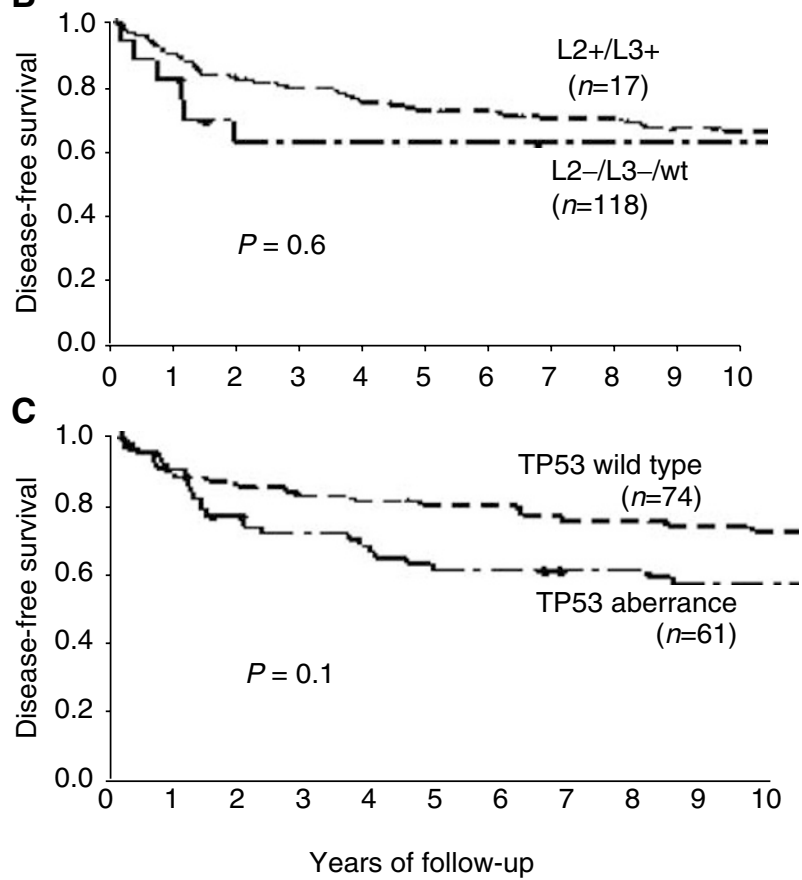

Figure 3 Disease-free survival related to TP53 alterations in exons 5-8 altering the protein. (A) TP53 mutations vs wild-type. (B) TP53 mutations affecting loop 2 and loop 3 of the protein $(\mathrm{L} 2+/ \mathrm{L} 3+)$ vs wild-type or other mutations (L2-/L3-/wt). (C) Aberrant TP53 scored as mutation and/or protein accumulation vs wild type.

Temporal temperature gradient gel electrophoresis is more sensitive and the result is rapid and easier to evaluate. Besides, the most common polymorphisms of the TP53 gene (codon 72 polymorphism in exon 4 and codon 213 polymorphism in exon 6) have specific patterns on TTGE gel, which are quite specific and are easy to distinguish by the naked eye. None of the eight mutations in exon 4 and the one mutation in exon 6 , which could not be confirmed by sequencing in the present study, had the patterns of the well-known polymorphisms on the TTGE gel. Thus, none of the samples with mutations listed in Table 1 under 'all exons' are likely to carry any of the common polymorphisms.

We found no mutational hotspots in the TP53 gene in human early-stage ovarian carcinomas, in agreement with previous investigators (Mazars et al, 1991; Okamoto et al, 1991; Meinhold-Heerlein et al, 2001). The most frequent mutations were found in codon 273 and in six samples, with one sample (No. 308) showing mutations in two of the bases in this codon. Whether these were two mutations on two different alleles or whether they resided on the same allele could not be decided. A frequent mutation in the IARC TP53 mutation database resides in codon 175. None of our samples showed this mutation. A control sample with this mutation was successfully analysed, confirming the negative result of this mutation in the described series. The codon 175 mutation might be a late event more common in advanced stages. Several novel mutations, not previously reported in the IARC TP53 mutation database in ovarian cancer, were determined. Otherwise, the spectrum of mutations is in agreement with that reported in the database.

Similar to the TP53 mutations reported in other types of human cancers, the majority (94.9\%) of TP53 mutations in the present study of early-stage ovarian carcinomas were point mutations. A low frequency of deletions was found in the present study, which is in agreement with a previous study (Wen et al, 1999) reporting deletions in 7\% after analysing all exons of the TP53 gene in 105 ovarian carcinomas.

In a review of TP53 mutations found in ovarian cancer (Skilling et al, 1996), it was reported that 234 of 280 mutations were nucleotide substitutions with 157 transitions and 77 transversions. Our finding of $75 \%$ transitions and $25 \%$ transversions is consistent with a high frequency of transitions in ovarian carcinoma, and the most common base substitution observed was G:C>A:T. The predominance of transition mutations suggests that TP53 mutations in ovarian carcinomas arise because of spontaneous errors in DNA synthesis and repair, rather than the direct interaction of carcinogens with DNA.

One of the features of the TP53 mutational spectra in human cancer is that transition at $\mathrm{CpG}$ dinucleotides contributes heavily to the mutation frequency in many cancers. However, in the present study, transitions at CpG sites were found only in $11(19.6 \%)$ of the 56 point mutations. This is significantly lower than that found among colon carcinomas (67\%) (Rodrigues et al, 1990), but is in agreement with previous observations that transitions at $\mathrm{CpG}$ dinucleotides are not frequent in ovarian cancer (Hollstein et al, 1991). This indicates that methylation of CpG sites and the level of spontaneous deamination may differ in various tissue types.

A few studies have described both TP53 mutation status and protein expression among ovarian carcinomas (Marks et al, 1991; Skilling et al, 1996; Wen et al, 1999). In the present study, we found TP53 protein accumulation in $32.6 \%$ of the cases. This is in accordance with the findings reported in the literature, which indicate increased protein expression in 26-69\% (Marks et al, 1991; Niwa et al, 1994; Skilling et al, 1996; Buttitta et al, 1997; Wen et al, 1999; Skirnisdottir et al, 2002). Although TP53 protein accumulation was significantly correlated with TP53 mutation in the present study, the concordance was only $71.1 \%$. The sensitivity, specificity, positive and negative predictive values of IHC in predicting for TP53 mutations were 56.4, 77.1, 50 and $81.3 \%$, respectively. Mutations usually lead to accumulation of mutated protein. However, some mutations create a stop codon and hence no protein is encoded, or some mutations do not lead to stable proteins that can be detected by IHC. Furthermore, mechanisms other than mutations may lead to accumulation like binding to cellular proteins or virus proteins or accumulation after a cellular response to DNA damage. One study (Hall et al, 1993) showed that a substantial part of the samples (39\%) without any detectable TP53 mutations may reveal increased TP53 protein levels by immunohistochemistry. This could reflect accumulation of normal TP53 protein induced by interacting proteins on a replicational, transcriptional or translational level. This means that IHC analysis of TP53 protein accumulation is a poor predictor of TP53 mutations, and accurate assessment of TP53 mutations requires DNA analyses.

Prognostic factors for patients with cancer are sought in order to learn about the natural progression of disease and to predict the outcome for individual patients. Additionally, these factors may help identify patients for whom the failure of conventional treatment could be predicted in advance. Although a number of authors (Dembo et al, 1990; Vergote et al, 2001) have evaluated traditional clinical parameters such as histology, grade, stage and presence of ascites as predictors for poor outcome in patients with stage I ovarian cancer, individual patient outcome is not entirely 
predictable based on these factors. Other clinical, pathological and molecular biological features have therefore been studied in an attempt to identify the additional factors of prognostic importance. Some studies have looked at the prognostic significance of TP53 alteration in ovarian cancers, and addressed the possibility that the clinical behaviour of tumours with TP53 alterations might be significantly different from that of tumours without TP53 alterations (Kohler et al, 1993; Kupryjanczyk et al, 1995; Geisler et al, 1997; Shahin et al, 2000; Fallows et al, 2001; Suzuki et al, 2001; Valverde et al, 2001). However, most of these studies addressed patients with advanced stage of the disease, and analysed relatively small numbers of tumours. In addition, TP53 protein accumulation has often been used as an indirect indicator of mutation. Therefore, diverging results have been reported on the prognostic significance of TP53 alterations in ovarian cancer, especially in early stage. Some studies have shown that TP53 alterations are more frequent in serous adenocarcinoma and in poorly differentiated tumours (Geisler et al, 1997; Leitao et al, 2002; Skirnisdottir et al, 2002), while others were not able to demonstrate these differences (Marks et al, 1991; Fallows et al, 2001; Leitao et al, 2002). Ploidy status has previously been demonstrated to be an independent prognostic factor among earlystage ovarian carcinomas (Vergote et al, 1993; Valverde et al, 2001). In the present study, TP53 protein accumulation was significantly related to DNA ploidy status, and was more often found in serous and unclassified adenocarcinomas as well as in poorly differentiated tumours. TP53 mutations were marginally less common in FIGO stage IA than in IB and IC; otherwise, no significant correlation was found to other clinicopathologic variables. Preliminary results from a series of advanced stage of ovarian carcinomas from our laboratory show a significant correlation to these parameters both for TP53 protein accumulation and TP53 mutations (unpublished data). These findings point to a role for TP53 mutations in progression of the disease, and that the changes in the TP53 gene in early stage are not sufficient for producing a highly malignant tumour.

In the present study, we have extensive follow-up of 178 patients with a median follow-up of 13.9 years. Although the presence of TP53 mutations did not seem to influence the disease-free survival significantly in early stage of ovarian carcinomas, patients with wild-type TP53 gene had around 15\% higher survival rate than patients with mutant TP53 gene after 5 years, which is in agreement with previous studies (Auer et al, 1996). The lack of statistical significance has to be evaluated on the basis of the number of the patients in the study. The different types of TP53 mutations, missense and nonmissense (nonsense, frameshift mutation and in-frame mutation) did not influence disease-free survival. TP53 mutations disrupting the loop 2 and loop 3 domains of the protein have been shown to predict poor survival in breast cancer (Borresen et al, 1995) and colorectal cancer (Borresen-Dale et al, 1998). Wen et al (1999) studied 105 ovarian cancers, and the overall survival for women with TP53 mutations in loop 2, loop 3 and loop-sheet-helix domains together showed a statistically significant difference in survival compared to survival of women whose ovarian cancers had other mutations. However, among these 105 patients, only 17 patients were in early stages. In the present study, the presence of TP53 mutations in loop 2 and loop 3 did not seem to influence the disease-free survival on early-stage disease, although a $17 \%$ difference in the 3 -year survival rate was seen. Combining TP53 mutation with protein accumulation has been found to be a stronger predictor of prognosis than TP53 mutation alone (Wen et al, 1999). This may indicate that tumour cells with TP53 accumulation without a mutation may have altered the TP53 pathway, resulting in a more aggressive behaviour. In the present study, we found a similar effect, but the difference in disease-free survival did not reach statistical significance.

Some studies have shown that loss of TP53 gene function may contribute to the drug resistance of ovarian cancer (Righetti et al, 1996; Buttitta et al, 1997; Marx et al, 1998). However, one study showed no significant association between TP53 mutation status and prognosis for patients who received platinum-based chemotherapy after analysing 45 FIGO stage IIC-III ovarian carcinomas (Smith-Sorensen et al, 1998). Platinum-based adjuvant chemotherapy was given to 59 out of 135 patients with complete TP53 mutation status from exons 5 to 8 after primary operation in the present study. However, TP53 mutation status had no significant influence on disease-free survival when this group was analysed separately. This disagreement could be explained by difference in stages between our study and previous studies (Righetti et al, 1996; Buttitta et al, 1997; Marx et al, 1998) and relative limited numbers of patients who were given platinum-based adjuvant chemotherapy after primary surgery in the present study.

\section{ACKNOWLEDGEMENTS}

We thank Johnsen $\mathrm{H}$ and $\mathrm{Vu} \mathrm{P}$ for their technical assistance with the TP53 mutation analyses. This project was supported by grants from the Norwegian Cancer Society and the Norwegian Research Council.

\section{REFERENCES}

Auer G, Einhorn N, Nilsson B, Silfversward C, Sjovall K (1996) Biological malignancy grading in early-stage ovarian carcinoma. Acta Oncol 35(Suppl 8): $93-98$

Bjorge T, Engeland A, Hansen S, Trope CG (1998) Prognosis of patients with ovarian cancer and borderline tumours diagnosed in Norway between 1954 and 1993. Int J Cancer 75: 663-670

Borresen AL, Andersen TI, Eyfjord JE, Cornelis RS, Thorlacius S, Borg A, Johansson U, Theillet C, Scherneck S, Hartman S (1995) TP53 mutations and breast cancer prognosis: particularly poor survival rates for cases with mutations in the zinc-binding domains. Genes Chromosomes Cancer 14: $71-75$

Borresen-Dale AL (1996) Constant Denaturant Gel Electrophoresis (CDGE) in mutation screening. In Technologies for detection of DNA damage and mutation, Pfeifer GP (ed) pp 267-279. New York: Plenum Press

Borresen-Dale AL (2003) TP53 and breast cancer. Hum Mutat 21: 292 - 300

Borresen-Dale AL, Lothe RA, Meling GI, Hainaut P, Rognum TO, Skovlund E (1998) TP53 and long-term prognosis in colorectal cancer: mutations in the L3 zinc-binding domain predict poor survival. Clin Cancer Res 4: $203-210$
Buller RE, Shahin MS, Holmes RW, Hatterman M, Kirby PA, Sood AK (2001) p53 Mutations and microsatellite instability in ovarian cancer: Yin and yang. Am J Obstet Gynecol 184: 891-902

Buttitta F, Marchetti A, Gadducci A, Pellegrini S, Morganti M, Carnicelli V, Cosio S, Gagetti O, Genazzani AR, Bevilacqua G (1997) p53 alterations are predictive of chemoresistance and aggressiveness in ovarian carcinomas: a molecular and immunohistochemical study. Br J Cancer 75: $230-235$

Dembo AJ, Davy M, Stenwig AE, Berle EJ, Bush RS, Kjorstad K (1990) Prognostic factors in patients with stage I epithelial ovarian cancer. Obstet Gynecol 75: $263-273$

Fallows S, Price J, Atkinson RJ, Johnston PG, Hickey I, Russell SE (2001) P53 mutation does not affect prognosis in ovarian epithelial malignancies. J Pathol 194: $68-75$

Geisler JP, Geisler HE, Wiemann MC, Givens SS, Zhou Z, Miller GA (1997) Quantification of p53 in epithelial ovarian cancer. Gynecol Oncol 66: $435-438$

Hall PA, McKee PH, Menage HD, Dover R, Lane DP (1993) High levels of p53 protein in UV-irradiated normal human skin. Oncogene 8: 203-207 
Hollstein M, Sidransky D, Vogelstein B, Harris CC (1991) p53 mutations in human cancers. Science 253: $49-53$

Hsu SM, Raine L, Fanger H (1981) A comparative study of the peroxidaseantiperoxidase method and an avidin-biotin complex method for studying polypeptide hormones with radioimmunoassay antibodies. Am J Clin Pathol 75: $734-738$

Iacopetta B (2003) TP53 mutation in colorectal cancer. Hum Mutat 21: $271-276$

Kohler MF, Kerns BJ, Humphrey PA, Marks JR, Bast Jr RC, Berchuck A (1993) Mutation and overexpression of p53 in early-stage epithelial ovarian cancer. Obstet Gynecol 81: 643-650

Kupryjanczyk J, Bell DA, Dimeo D, Beauchamp R, Thor AD, Yandell DW (1995) p53 gene analysis of ovarian borderline tumors and stage I carcinomas. Hum Pathol 26: $387-392$

Kupryjanczyk J, Thor AD, Beauchamp R, Merritt V, Edgerton SM, Bell DA, Yandell DW (1993) p53 gene mutations and protein accumulation in human ovarian cancer. Proc Natl Acad Sci USA 90: 4961 - 4965

Leitao MM, Soslow R, Olvera N, Arroyo C, Baergen R, Boyd J (2002) TP53 mutation in early stage sporadic epithelial ovarian carcinoma. Proc Annu Meet Am Soc Clin Oncol J Clin Oncol 21: A818

Levine AJ, Momand J, Finlay CA (1991) The p53 tumour suppressor gene. Nature 351: $453-456$

Marks JR, Davidoff AM, Kerns BJ, Humphrey PA, Pence JC, Dodge RK, Clarke-Pearson DL, Iglehart JD, Bast Jr RC, Berchuck A (1991) Overexpression and mutation of p53 in epithelial ovarian cancer. Cancer Res 51: 2979-2984

Marx D, Meden H, Ziemek T, Lenthe T, Kuhn W, Schauer A (1998) Expression of the p53 tumour suppressor gene as a prognostic marker in platinum-treated patients with ovarian cancer. Eur J Cancer 34: $845-850$

Mazars R, Pujol P, Maudelonde T, Jeanteur P, Theillet C (1991) p53 mutations in ovarian cancer: a late event? Oncogene 6: 1685-1690

Meinhold-Heerlein I, Ninci E, Ikenberg H, Brandstetter T, Ihling C, Schwenk I, Straub A, Schmitt B, Bettendorf H, Iggo R, Bauknecht T (2001) Evaluation of methods to detect p53 mutations in ovarian cancer. Oncology 60: 176-188

Niwa $\mathrm{K}$, Itoh $\mathrm{M}$, Murase $\mathrm{T}$, Morishita $\mathrm{S}$, Itoh $\mathrm{N}$, Mori $\mathrm{H}$, Tamaya $\mathrm{T}$ (1994) Alteration of p53 gene in ovarian carcinoma: clinicopathological correlation and prognostic significance. $\mathrm{Br} J$ Cancer 70: $1191-1197$

Okamoto A, Sameshima Y, Yokoyama S, Terashima Y, Sugimura T, Terada M, Yokota J (1991) Frequent allelic losses and mutations of the p53 gene in human ovarian cancer. Cancer Res 51: $5171-5176$

Reles A, Wen WH, Schmider A, Gee C, Runnebaum IB, Kilian U, Jones LA, El Naggar A, Minguillon C, Schonborn I, Reich O, Kreienberg R, Lichtenegger W, Press MF (2001) Correlation of p53 mutations with resistance to platinum-based chemotherapy and shortened survival in ovarian cancer. Clin Cancer Res 7: 2984-2997

Righetti SC, Della TG, Pilotti S, Menard S, Ottone F, Colnaghi MI, Pierotti MA, Lavarino C, Cornarotti M, Oriana S, Bohm S, Bresciani GL, Spatti G, Zunino F (1996) A comparative study of p53 gene mutations, protein accumulation, and response to cisplatin-based chemotherapy in advanced ovarian carcinoma. Cancer Res 56: 689-693

Rodrigues NR, Rowan A, Smith ME, Kerr IB, Bodmer WF, Gannon JV, Lane DP (1990) p53 mutations in colorectal cancer. Proc Natl Acad Sci USA 87: $7555-7559$

Schildkraut JM, Halabi S, Bastos E, Marchbanks PA, McDonald JA, Berchuck A (2000) Prognostic factors in early-onset epithelial ovarian cancer: a population-based study. Obstet Gynecol 95: 119-127

Shahin MS, Hughes JH, Sood AK, Buller RE (2000) The prognostic significance of p53 tumor suppressor gene alterations in ovarian carcinoma. Cancer 89: 2006-2017

Skilling JS, Sood A, Niemann T, Lager DJ, Buller RE (1996) An abundance of p53 null mutations in ovarian carcinoma. Oncogene 13: 117-123

Skirnisdottir I, Seidal T, Gerdin E, Sorbe B (2002) The prognostic importance of $\mathrm{p} 53, \mathrm{bcl}-2$, and bax in early stage epithelial ovarian carcinoma treated with adjuvant chemotherapy. Int J Gynecol Cancer 12: $265-276$

Smith-Sorensen B, Kaern J, Holm R, Dorum A, Trope C, Borresen-Dale AL (1998) Therapy effect of either paclitaxel or cyclophosphamide combination treatment in patients with epithelial ovarian cancer and relation to TP53 gene status. $\mathrm{Br} J$ Cancer 78: 375-381

Sood AK, Sorosky JI, Dolan M, Anderson B, Buller RE (1999) Distant metastases in ovarian cancer: association with p53 mutations. Clin Cancer Res 5: $2485-2490$

Sudbo J, Kildal W, Risberg B, Koppang HS, Danielsen HE, Reith A (2001) DNA content as a prognostic marker in patients with oral leukoplakia. $N$ Engl J Med 344: 1270-1278

Suzuki M, Ohwada M, Saga Y, Kohno T, Takei Y, Sato I (2001) Micrometastatic p53-positive cells in the lymph nodes of early stage epithelial ovarian cancer: prognostic significance. Oncology 60: 170-175

Sørlie T, Vu P, Johnsen H, Lind GE, Lothe RA, Børresen-Dale A-L (2003) Protocol for mutation screening of the TP53 gene by temperature gel electrophoresis (TTGE). In Methods in Molecular Biology, Methods for Detecting and Characterizing Genetic Alterations. Clifton, UK: Humana Press

Valverde JJ, Martin M, Garcia-Asenjo JA, Casado A, Vidart JA, Diaz-Rubio E (2001) Prognostic value of DNA quantification in early epithelial ovarian carcinoma. Obstet Gynecol 97: 409-416

Vergote I, De Brabanter J, Fyles A, Bertelsen K, Einhorn N, Sevelda P, Gore ME, Kaern J, Verrelst H, Sjovall K, Timmerman D, Vandewalle J, Van Gramberen M, Trope CG (2001) Prognostic importance of degree of differentiation and cyst rupture in stage I invasive epithelial ovarian carcinoma. Lancet 357: 176-182

Vergote IB, Kaern J, Abeler VM, Pettersen EO, De Vos LN, Trope CG (1993) Analysis of prognostic factors in stage I epithelial ovarian carcinoma: importance of degree of differentiation and deoxyribonucleic acid ploidy in predicting relapse. Am J Obstet Gynecol 169: 40-52

Wen WH, Reles A, Runnebaum IB, Sullivan-Halley J, Bernstein L, Jones LA, Felix JC, Kreienberg R, El Naggar A, Press MF (1999) p53 mutations and expression in ovarian cancers: correlation with overall survival. Int $J$ Gynecol Pathol 18: 29-41 